\title{
Design and analysis of low frequency strut-straddling feed arrays for EVLA reflector antennas
}

\author{
M. Harun ${ }^{1}$ and S. W. Ellingson ${ }^{1}$ \\ Received 15 March 2011; revised 18 July 2011; accepted 24 July 2011; published 18 October 2011.
}

[1] A new feed system is designed for operation below $100 \mathrm{MHz}$. The only existing system on the EVLA operating below $100 \mathrm{MHz}$ is the "4 m" (74 MHz) system which uses crossed half-wave dipoles located in front of the Cassegrain subreflector as the feed. However, the dipole feeds of this system introduce blockage, and a reduction in system sensitivity (estimated to be $\sim 6 \%$ at $1.4 \mathrm{GHz}$ ) is observed at higher frequency bands; hence the dipoles are removed most of the time. An alternative feed concept is therefore proposed in this paper. The proposed system appears to reduce sensitivity degradation at $1.4 \mathrm{GHz}$ by $3 \%$ and thus might be permanently mounted. Moreover, the new system has sensitivity comparable to the existing system at frequencies below $100 \mathrm{MHz}$. The feed for this system consists of dipoles mounted between the adjacent struts of the reflector and is thus referred to as a strut-straddling feed array. This design and the analysis methodology used in this paper should be applicable in meeting the contiguous frequency coverage requirement (50-470 MHz) of the new low frequency system proposed for the EVLA. Also, it may be applied in the modification of other existing large reflector antennas for low frequency operation.

Citation: Harun, M., and S. W. Ellingson (2011), Design and analysis of low frequency strut-straddling feed arrays for EVLA reflector antennas, Radio Sci., 46, RS0M04, doi:10.1029/2011RS004710.

\section{Introduction}

[2] The Expanded Very Large Array (EVLA) consists of 27 Cassegrain reflector antennas on the Plains of San Agustin, New Mexico. Each reflector antenna is 25 meters in diameter, and has a focal length $(f)$ to diameter $(D)$ ratio of 0.36. An EVLA reflector antenna is shown in Figure 1. The original design included capabilities at four bands corresponding to wavelengths of $21 \mathrm{~cm}(\approx 1.4 \mathrm{GHz}), 6 \mathrm{~cm}$ (5 GHz), $2 \mathrm{~cm}(15 \mathrm{GHz})$, and $1.3 \mathrm{~cm}(23 \mathrm{GHz})$ [Napier et al., 1983]. The first low frequency system, operating at $90 \mathrm{~cm}$, was installed in 1989. This system employed crossed dipoles mounted in front of the Cassegrain subreflector as the feed. The success of the $90 \mathrm{~cm}$ system led to the consideration of a lower-frequency capability. The " $4 \mathrm{~m}$ " system operating at $74 \mathrm{MHz}$ was thus installed, and is described by Kassim et al. [1993, 2007]. The feed for this system also consists of crossed half-wave dipoles in front of the subreflector, and yields an aperture efficiency of somewhere in the range 15\% [Kassim et al., 1993] to 25\% [Kassim et al., 2007]. Blockage and scattering from the $4 \mathrm{~m}$ system reduces the sensitivity of the $21 \mathrm{~cm}$ system by about $6 \%$ [Kassim et al., 1993]; thus, the dipoles are not permanently installed and are only intermittently used.

\footnotetext{
${ }^{1}$ Bradley Department of Electrical and Computer Engineering, Virginia Tech, Blacksburg, Virginia, USA.

Copyright 2011 by the American Geophysical Union. 0048-6604/11/2011RS004710
}

[3] A new "low frequency system" (LFS) with the goal of covering 50-470 $\mathrm{MHz}$ has been proposed for the EVLA [Ott et al., 2009]. Additional feeds will probably be required to accommodate this frequency range. In this paper, we focus our interest in covering the frequency range below $100 \mathrm{MHz}$. Traditional approaches to feed design for covering large tuning ranges focus on achieving large impedance bandwidth which results in large, complex feeds, e.g., the Eleven feed [Olsson et al., 2006]. However, in the frequency regime of our interest, simple dipole-like antennas can offer large usable bandwidth from a sensitivity perspective. This is because at these frequencies, Galactic noise can easily dominate over the self-noise of the electronics connected to the antenna. Once the system is Galactic noiselimited, improving the impedance matching between the antenna and the connected electronics does not significantly improve sensitivity [Ellingson, 2005]. As a result, the usable bandwidth for a dipole-like antenna under these conditions tends to be much larger than its narrow impedance bandwidth. For example, Ellingson et al. [2007] show that a $38 \mathrm{MHz}$ resonant dipole with a $300 \mathrm{~K}$ front end achieves Galactic noise-limited sensitivity over the range of 29 $47 \mathrm{MHz}$ at least.

[4] The notion of low frequency feeds for large reflector antennas is not new; however prior work did not allow simultaneous operation with L-band and higher-frequency systems. For example, the $50 \mathrm{MHz}$ system employed on the GMRT employs dipoles as a low frequency feed; this feed is co-located with the existing $327 \mathrm{MHz}$ feed. However, the $50-$ and $327-\mathrm{MHz}$ dipoles are located above a $3 \mathrm{~m} \times 3 \mathrm{~m}$ 


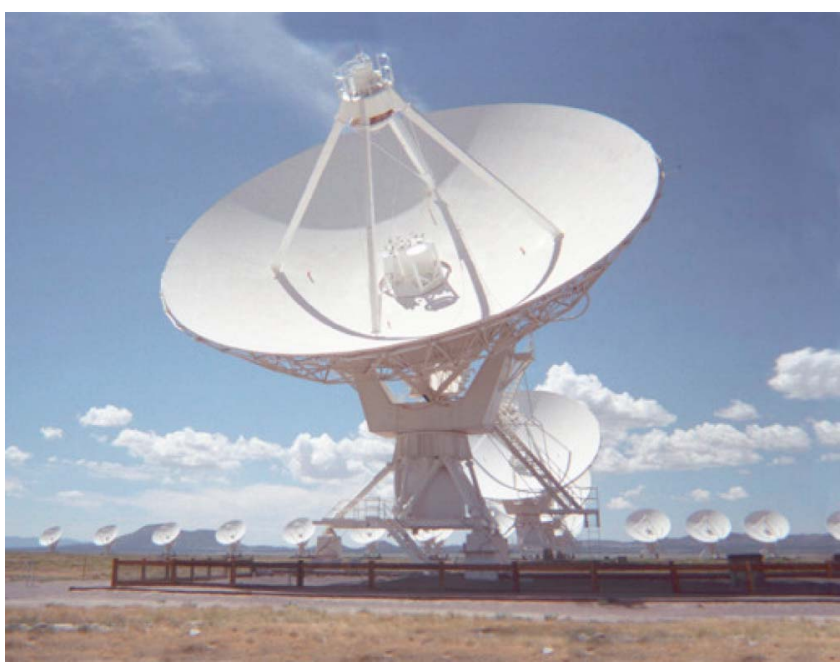

Figure 1. An EVLA reflector antenna. Image source is NRAO/AUI, P. A. Lofy, Photographer. Available at http:// images.nrao.edu/Telescopes/VLA/480.

square reflector which must be rotated out of position in order for higher frequencies to be used [Shankar et al., 2009]. A low frequency system employing retractable folded dipole feeds was briefly installed on the WSRT for operation from 110 to $190 \mathrm{MHz}$ [van der Marel et al., 2005].

[5] In this paper, we first characterize the performance of the existing EVLA $4 \mathrm{~m}$ system in the range 50-88 $\mathrm{MHz}$.
Then, in order to resolve the blockage issue associated with the existing system, we propose a new feed consisting of a ring of dipoles mounted between adjacent struts. This arrangement is referred to as a strut-straddling feed array. The motivation behind this design is the fact that, at low frequencies, the available power in the volume in front of a reflector spreads out from the focal region. As an example, the distribution of power density (co-pol) in the focal plane of a simple $25 \mathrm{~m}$ diameter paraboloidal reflector upon a plane wave incidence at $500 \mathrm{MHz}$ and $50 \mathrm{MHz}$ are shown in Figures 2 and 3, respectively. It can be seen that the available power spreads out significantly as the frequency is lowered from $500 \mathrm{MHz}$ to $50 \mathrm{MHz}$. As a result, even if we move about $2 \mathrm{~m}$ away from the focus, the amount of available power drops only by about half at $50 \mathrm{MHz}$. This suggests the possibility of absorbing an appreciable amount of power by employing an array of feeds away from the focal region, and appropriately combining the signals from these feeds. Based on this philosophy, we show in this paper that the new system offers sensitivity comparable to the EVLA 4 m system in the frequency regime below $100 \mathrm{MHz}$, but with reduced blockage ( $\sim 3 \%$ improvement) at higher frequency bands.

[6] The rest of this paper is organized as follows. Section 2 describes the methodology used to analyze the sensitivity of a reflector antenna. Sensitivity is expressed in terms of the system equivalent flux density (SEFD), which is defined as the flux spectral density $\left(\mathrm{W} \mathrm{m}^{-2} \mathrm{~Hz}^{-1}\right)$ which yields signalto-noise ratio (SNR) of unity at the output of the system. Section 3 describes the electromagnetic modeling of an EVLA reflector antenna required for analysis using the

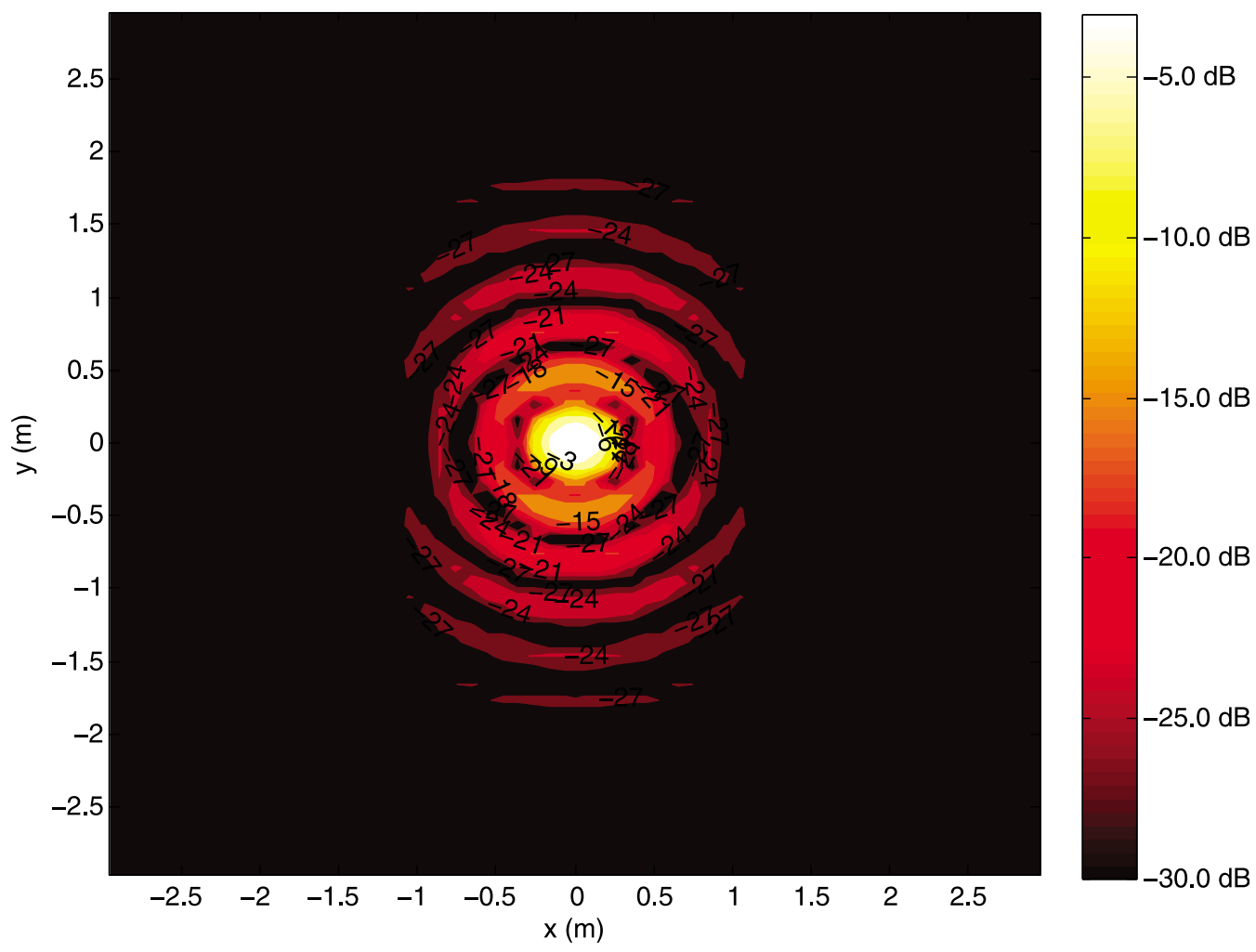

Figure 2. Distribution of power density in the focal plane of a reflecting paraboloid $(D=25 \mathrm{~m}$, $f / D=0.36$ ) relative to the power density at the focus at $500 \mathrm{MHz}$. 


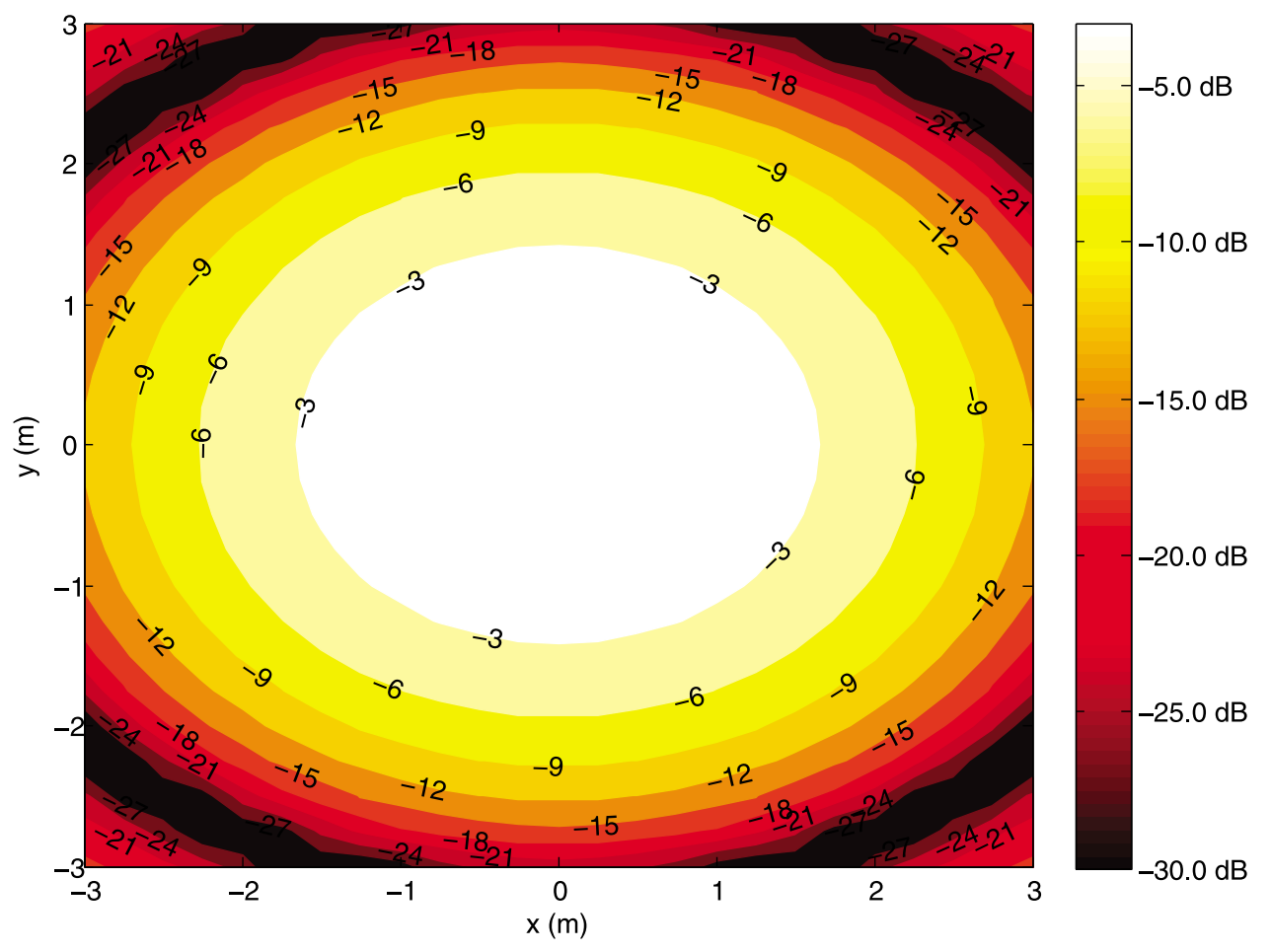

Figure 3. Distribution of power density in the focal plane of a reflecting paraboloid $(D=25 \mathrm{~m}$, $f / D=0.36$ ) relative to the power density at the focus at $50 \mathrm{MHz}$.

method of moments (MoM). The findings of sections 2 and 3 are then applied in section 4 to estimate the sensitivity of the existing EVLA $4 \mathrm{~m}$ system. An analysis of sensitivity of the EVLA $4 \mathrm{~m}$ system as presented in this section has not been previously reported to the best of our knowledge. Section 5 describes the strut-straddling feed array design, and compares the sensitivity and associated blockage of the new system with the existing system. Conclusions are summarized in section 6 .

\section{Methodology for Analysis of the Sensitivity of Dipole Arrays Used as a Reflector Feed}

[7] The metric used in this paper for evaluating the performance of low frequency systems is sensitivity. Sensitivity is expressed in terms of SEFD. SEFD is a useful metric as it includes the combined effect of the feed antennas and all sources of noise into a single number that is directly related to the sensitivity of astronomical observations. A detailed formulation of SEFD for analyzing low frequency systems is presented in this section. An important aspect of this formulation is that the correlation of external noise between the array elements is not ignored. The correlation of external noise can significantly desensitize the system [Ellingson, 2011]. This is more of a concern at low frequencies where the external noise can easily dominate the internal noise of the system. Therefore, the particular formulation of SEFD presented in this paper is different from most other formulations, and feeds our purpose of evaluating low frequency systems. Part of the formulation provided in this section is given by Ellingson [2011]; however, it is still presented here briefly, since some of the intermediate results are used for the analysis.
[8] Let $E_{\theta}(t)$ and $E_{\phi}(t)$ be the $\theta$ - and $\phi$-polarized components of the electric field associated with the signal of our interest, incident from a direction of $\left\{\theta_{0}, \phi_{0}\right\}$, having units of $\mathrm{V} \mathrm{m}^{-1} \mathrm{~Hz}^{-1 / 2}$. The direction $\left\{\theta_{0}, \phi_{0}\right\}$ will be represented as $\psi_{0}$ henceforth. The resulting voltage at the terminals of the $n$th feed antenna, having units of $\mathrm{V} \mathrm{Hz}^{-1 / 2}$, is

$$
x_{n}(t)=a_{n}^{\theta}\left(\psi_{0}\right) E_{\theta}(t)+a_{n}^{\phi}\left(\psi_{0}\right) E_{\phi}(t)+z_{n}(t)+u_{n}(t)
$$

where $a_{n}^{\theta}\left(\psi_{0}\right)$ and $a_{n}^{\phi}\left(\psi_{0}\right)$ are the effective lengths, having units of meters, associated with the $\theta$ and $\phi$ polarizations respectively, for the $n$th feed antenna for a plane wave incident from $\psi_{0} ; z_{n}(t)$ is the contribution from noise external to the system; and $u_{n}(t)$ is the contribution from noise internal to the system. When the signals from all the feed antennas are combined, the output can be expressed as

$$
y(t)=\sum_{n=1}^{N} b_{n} x_{n}(t)
$$

where $N$ is the number of feed antennas, and the unitless $b_{n}$ 's are the combining coefficients. In this paper, we assume each dipole is connected to a receive path that is fully characterized in terms of its input impedance, $R_{L}$, and its input-referred noise temperature, $T_{p}$. One way this could be realized is to use an "active balun" which presents a differential input impedance of $R_{L}$ to the dipole, a single-ended output to a coaxial cable, and provides gain sufficient to dominate the input-referred noise temperature. This scheme is described by Ellingson et al. [2007, 2009] and Ellingson [2011]. The beam-forming coefficients can be implemented 
by varying the length or phase of the cables before an analog combiner, or the signals from the cables can be digitized individually and combined digitally.

[9] Assuming root-mean square voltages, the available power at the output of the combiner is

$$
P_{y}=\left\langle y(t) y^{*}(t)\right\rangle R_{0}^{-1}
$$

where angle brackets denote time domain averaging, asterisk denotes conjugation and $R_{0}$ is the impedance looking into the system as seen from the terminals across which $y(t)$ is measured. We will now expand equation (3) with the assumptions that the signal of interest, $z_{n}(t)$ and $u_{n}(t)$ are mutually uncorrelated for any given $n$; i.e., for any $n$ and $m$,

$$
\begin{gathered}
\left\langle E_{\theta}(t) z_{n}^{*}(t)\right\rangle=\left\langle E_{\phi}(t) z_{n}^{*}(t)\right\rangle=0 \\
\left\langle E_{\theta}(t) u_{n}^{*}(t)\right\rangle=\left\langle E_{\phi}(t) u_{n}^{*}(t)\right\rangle=0 \text { and } \\
\left\langle z_{n}(t) u_{m}^{*}(t)\right\rangle=0
\end{gathered}
$$

The correlation of external noise between antennas is not precluded by the above assumption since $\left\langle z_{n}(t) z_{m}^{*}(t)\right\rangle$ can be $\neq 0$ for $n \neq m$. Furthermore no assumption has been made about the correlation between $E_{\theta}(t)$ and $E_{\phi}(t)$. Upon expanding equation (3) we obtain

$$
\begin{aligned}
P_{y} R_{o}= & \mathbf{b}^{H} \mathbf{A}_{\theta \theta} \mathbf{b} P_{\theta \theta}+\mathbf{b}^{H} \mathbf{A}_{\phi \phi} \mathbf{b} P_{\phi \phi}+\mathbf{b}^{H} \mathbf{A}_{\theta \phi} \mathbf{b} P_{\theta \phi}+\mathbf{b}^{H} \mathbf{A}_{\phi \theta} \mathbf{b} P_{\phi \theta} \\
& +\mathbf{b}^{H} \mathbf{P}_{z} \mathbf{b}+\mathbf{b}^{H} \mathbf{P}_{u} \mathbf{b}
\end{aligned}
$$

where " $H$ " denotes the conjugate transpose operator;

$$
\mathbf{b}=\left[\begin{array}{llll}
b_{1} & b_{2} & . . & b_{N}
\end{array}\right]^{T}
$$

where superscript $T$ denotes the transpose operator; and

$$
\begin{gathered}
\mathbf{A}_{\theta \theta}=\mathbf{a}_{\theta}^{*}\left(\psi_{o}\right) \mathbf{a}_{\theta}^{T}\left(\psi_{o}\right) P_{\theta \theta}=\left\langle\left|E_{\theta}(t)\right|^{2}\right\rangle \\
\mathbf{A}_{\phi \phi}=\mathbf{a}_{\phi}^{*}\left(\psi_{o}\right) \mathbf{a}_{\phi}^{T}\left(\psi_{o}\right) P_{\phi \phi}=\left\langle\left|E_{\phi}(t)\right|^{2}\right\rangle \\
\mathbf{A}_{\theta \phi}=\mathbf{a}_{\phi}^{*}\left(\psi_{o}\right) \mathbf{a}_{\theta}^{T}\left(\psi_{o}\right) P_{\theta \phi}=\left\langle E_{\theta}(t) E_{\phi}^{*}(t)\right\rangle \\
\mathbf{a}_{\phi \theta}=\mathbf{a}_{\theta}^{*}\left(\psi_{o}\right) \mathbf{a}_{\phi}^{T}\left(\psi_{o}\right) P_{\phi \theta}=\left\langle E_{\phi}(t) E_{\theta}^{*}(t)\right\rangle \\
\mathbf{a}_{\theta}\left(\psi_{o}\right)=\left[a_{1}^{\theta}\left(\psi_{o}\right) a_{2}^{\theta}\left(\psi_{o}\right) \ldots a_{N}^{\theta}\left(\psi_{o}\right)\right]^{T} \text { and } \\
\mathbf{a}_{\phi}\left(\psi_{o}\right)=\left[a_{1}^{\phi}\left(\psi_{o}\right) a_{2}^{\phi}\left(\psi_{o}\right) \ldots a_{N}^{\phi}\left(\psi_{o}\right)\right]^{T}
\end{gathered}
$$

However, for an unpolarized signal of interest $P_{\theta \phi}=P_{\phi \theta}=0$ and $P_{\theta \theta}=P_{\phi \phi}=\eta S(\psi) / 2$, where $S(\psi)$ is the flux density associated with the signal.

[10] $\mathbf{P}_{z}$ in equation (7) is a matrix whose $(n, m)$ th element is $\left\langle z_{n}^{*}(t) z_{m}(t)\right\rangle$, and $\mathbf{P}_{u}$ is a matrix whose $(n, m)$ th element is $\left\langle u_{n}^{*}(t) u_{m}(t)\right\rangle$. We now find a simple expression for the $(n, m)$ th element of $\mathbf{P}_{z}$. The power in two polarizations of the electric field associated with the unpolarized external noise incident from a region of solid angle $\Delta \Omega$ around $\psi$ can be expressed as

$$
\left\langle\left|\Delta E_{\theta}(\psi, t)\right|^{2}\right\rangle=\left\langle\left|\Delta E_{\phi}(\psi, t)\right|^{2}\right\rangle=\frac{\eta}{2} \Delta S(\psi)
$$

where $\eta$ is impedance of free space and $\Delta S(\psi)$ is the associated flux density having units of $\mathrm{W} \mathrm{m}^{-2} \mathrm{~Hz}^{-1} . \Delta S(\psi)$ can be obtained from Rayleigh-Jeans Law:

$$
\Delta S(\psi)=\frac{2 k}{\lambda^{2}} T_{b}(\psi) \Delta \Omega
$$

where $T_{b}(\psi)$ is the brightness temperature in the direction $\psi$, and $\lambda$ is wavelength. We can thus model $\Delta E_{\theta}(\psi, t)$ and $\Delta E_{\phi}(\psi, t)$ as follows:

$$
\begin{aligned}
& \Delta E_{\theta}(\psi, t)=g_{\theta}(\psi, t) \sqrt{\frac{k \eta}{\lambda^{2}} T_{b}(\psi) \Delta \Omega} \\
& \Delta E_{\phi}(\psi, t)=g_{\phi}(\psi, t) \sqrt{\frac{k \eta}{\lambda^{2}} T_{b}(\psi) \Delta \Omega}
\end{aligned}
$$

where $g_{\theta}(\psi, t)$ and $g_{\phi}(\psi, t)$ are Gaussian-distributed independent random variables with zero mean and unit variance. Now, in order to obtain $z_{n}(t)$ we sum the contributions received over a sphere:

$$
z_{n}(t)=\sum_{\psi}\left[a_{n}^{\theta}(\psi) \Delta E_{\theta}(\psi, t)+a_{n}^{\phi}(\psi) \Delta E_{\phi}(\psi, t)\right]
$$

Applying the definition of $\mathbf{P}_{z}$ and exploiting the statistical properties of $g_{\theta}(\psi, t)$ and $g_{\phi}(\psi, t)$ we find

$$
\mathbf{P}_{z}^{[n, m]}=\frac{k \eta}{\lambda^{2}} \sum_{\psi}\left[a_{n}^{\theta^{*}}(\psi) a_{m}^{\theta}(\psi)+a_{n}^{\phi^{*}}(\psi) a_{m}^{\phi}(\psi)\right] T_{b}(\psi) \Delta \Omega
$$

Returning to equation (7), we obtain the following expression for the signal-to-noise ratio (SNR) for an unpolarized signal of interest:

$$
\mathrm{SNR}=\frac{\eta}{2} S(\psi) \frac{\mathbf{b}^{H}\left(\mathbf{A}_{\theta \theta}+\mathbf{A}_{\phi \phi}\right) \mathbf{b}}{\mathbf{b}^{H}\left(\mathbf{P}_{z}+\mathbf{P}_{u}\right) \mathbf{b}}
$$

The maximum possible SNR is equal to the maximum eigenvalue of $\mathbf{R}_{n}^{-1} \mathbf{R}_{s}$ [Monzingo and Miller, 1980], where

$$
\begin{gathered}
\mathbf{R}_{n}=\mathbf{P}_{z}+\mathbf{P}_{u} \text { and } \\
\mathbf{R}_{s}=\mathbf{A}_{\theta \theta} P_{\theta \theta}+\mathbf{A}_{\phi \phi} P_{\phi \phi}
\end{gathered}
$$

The maximum SNR is achieved by selecting $\mathbf{b}$ to be the eigenvector corresponding to the maximum eigenvalue of $\mathbf{R}_{n}^{-1} \mathbf{R}_{s}$. To summarize, the procedure to compute the optimal beam-forming cofficients $\left(b_{n}\right.$ 's) is as follows: (1) compute $\mathbf{R}_{s}$ using equations (9), (10) and (23); (2) compute $\mathbf{R}_{n}$ using equation (22), and (3) compute $\mathbf{b}=\mathbf{R}_{n}^{-1} \mathbf{R}_{s}$. Note that $P_{\theta \theta}$ and $P_{\phi \phi}$ may be taken to be unity for simplicity, since these values merely scale $\mathbf{b}$, which has no impact on SNR. $\mathbf{P}_{z}$ in 
equation (22) may be obtained either by direct measurement (using the definition of $\mathbf{P}_{z}$ ), or by simulation as implied by equation (20). Similarly, $\mathbf{P}_{u}$ in equation (22) can be obtained either by measurement or from advance knowledge of the receiver temperatures.

[11] Regardless of the choice of $\mathbf{b}$, the sensitivity of the antenna system can now be expressed in terms of SEFD, which is the value of $S(\psi)$ that gives an SNR of 1; i.e.,

$$
\mathrm{SEFD}=\frac{2}{\eta} \frac{\mathbf{b}^{H}\left(\mathbf{P}_{z}+\mathbf{P}_{u}\right) \mathbf{b}}{\mathbf{b}^{H}\left(\mathbf{A}_{\theta \theta}+\mathbf{A}_{\phi \phi}\right) \mathbf{b}}
$$

Note that this expression gives SEFD directly in terms of feed element effective lengths (i.e., gains), external and internal noise powers, and the combining coefficients.

\section{Electromagnetic Modeling of an EVLA Reflector Antenna}

[12] In this section, we describe an electromagnetic model of an EVLA reflector antenna. In our frequency range of interest, the reflector is not very large compared to a wavelength, and therefore requires "full-wave" analysis (e.g., MoM) for accurate results. In this paper, we employ the NEC2 implementation of MoM [Burke and Poggio, 1981] for this purpose.

[13] The major parts of an EVLA reflector antenna are: main reflector, subreflector, struts, support wires, subreflector mount, and high-frequency feed assembly. Figure 4 shows a drawing of these components (minus the main reflector) along with their dimensions. Study shows that all these components have impact on the gain and pattern of the reflector antenna [Harun and Ellingson, 2008]. Therefore, an accurate model of an EVLA antenna should include all of these components.

[14] The main reflector is modeled as a wire grid. The grid spacing (smallest distance between two parallel wires in the grid) and the wire radius are selected based on a simple experiment. A wire grid model of a flat disk of diameter $25 \mathrm{~m}$ is used as a ground plane, and a half-wave dipole is located quarter wavelength (at $74 \mathrm{MHz}$ ) above this grid. The grid spacing and wire radius are then varied to achieve a performance (gain and pattern) which is comparable to the performance observed when the grid is replaced by a perfect solid ground plane. Experiments showed that a wire grid with grid spacing of $36.5 \mathrm{~cm}$ and wire radius of $45 \mathrm{~mm}$ achieves this criterion while using a manageable number of segments.

[15] In the model used in this paper, the main reflector is centered around the $z$-axis of the coordinate system with its circumference parallel to the $x y$-plane, and the prime focus coincides with the origin of the coordinate system. We will refer to this coordinate system in the rest of this paper for identifying different locations in the antenna model. The main reflector model consists of the wire grid (as determined in the previous paragraph) paraboloid with $f / D=0.36$, and $D=25 \mathrm{~m}$.

[16] The subreflector has a diameter of $2.35 \mathrm{~m}$. Its shape is not known to us in detail; however a drawing of the subreflector was made available to us. Using the drawing we extracted data points along the cross section of the subreflector and used a polynomial equation to fit the data within a residual (difference between the data point and the predicted value from the polynomial equation) of $0.01 \lambda$ at $74 \mathrm{MHz}$. From this we created a wire grid model for the subreflector in the same manner as the main reflector. We did not use the same grid spacing as the main reflector, as this spacing is very large with respect to the diameter of the subreflector, and results in excessively large "quantization error" in the rim of the subreflector. Therefore, we used a smaller grid spacing of $12 \mathrm{~cm}$ to reduce the error. Also, in the complete antenna model the subreflector is not symmetrical about the axis of the reflector; it is slightly tilted. This is because in the actual EVLA antenna the subreflector is tilted in order to point toward feeds arranged in a ring near the vertex of the main reflector.

[17] The largest dimension of the cross section of a strut is $40 \mathrm{~cm}$, which at $74 \mathrm{MHz}$ is $0.1 \lambda$. We could model the strut as a single wire according to the Equal Area Rule [Rubinstein et al., 2005], but in our case the radius of the wire would be $10 \mathrm{~cm}$, which is larger than $\lambda /(20 \pi)$ at $74 \mathrm{MHz}$ and therefore not appropriate according to the modeling guidelines of NEC2. Instead, we model the strut as two wires of radius $3 \mathrm{~cm}$ placed $40 \mathrm{~cm}$ apart, with connecting segments every $50 \mathrm{~cm}$.

[18] The four support wires are modeled using single wires of radius $1 \mathrm{~cm}$, having segment lengths $\approx \lambda / 10$ at $74 \mathrm{MHz}$.

[19] The subreflector mount is modeled as a wire grid cylinder (visible in Figure 5). The top and bottom surfaces of the cylinder are modeled using concentric rings separated by a distance of $\lambda / 10$ at $74 \mathrm{MHz}$. Radial wires with segment lengths of $\lambda / 10$ are then created to connect the concentric rings. As for the cylindrical surface, two more circular rings are created in between the top and the bottom surfaces. These rings are then connected to the top and the bottom surfaces, and to each other using vertical wires of segment length $\lambda / 10$.

[20] The high-frequency feed assembly at the center of the main reflector is modeled as a wire grid rectangular box. The grid spacing and wire radius of this rectangular box is the same as that used for modeling the main reflector.

[21] We modeled the dipoles of the existing $4 \mathrm{~m}$ system using single wires having 11 segments. The radius of each of the dipoles is $2.38 \mathrm{~mm}$, and the length is selected to be $1.94 \mathrm{~m}$ to make it resonate at $74 \mathrm{MHz}$.

[22] The wire grid model explained above was developed so that the total number of segments at $74 \mathrm{MHz}$ is less than 11,000 and can fit in 2 GB of RAM. However, the model meets the NEC2 modeling guidelines up to $100 \mathrm{MHz}$.

[23] In order to check the validity of the model we analyze the gain and pattern of the EVLA $4 \mathrm{~m}$ system at $74 \mathrm{MHz}$. In this analysis, only one dipole feed is considered at a time. The dipole is located at the image (as reflected by the subreflector) of the prime focus. The support wires may be oriented in two different ways with respect to a dipole feed, as shown in Figures 5 and 6, and have different impacts on the gain and the pattern. One of these orientations is when the wires are roughly aligned with the dipole; this will be referred to as the co-aligned orientation (Figure 5). The other orientation is when the wires are roughly cross-aligned with the dipole; this will be referred to as the cross-aligned orientation (Figure 6). 


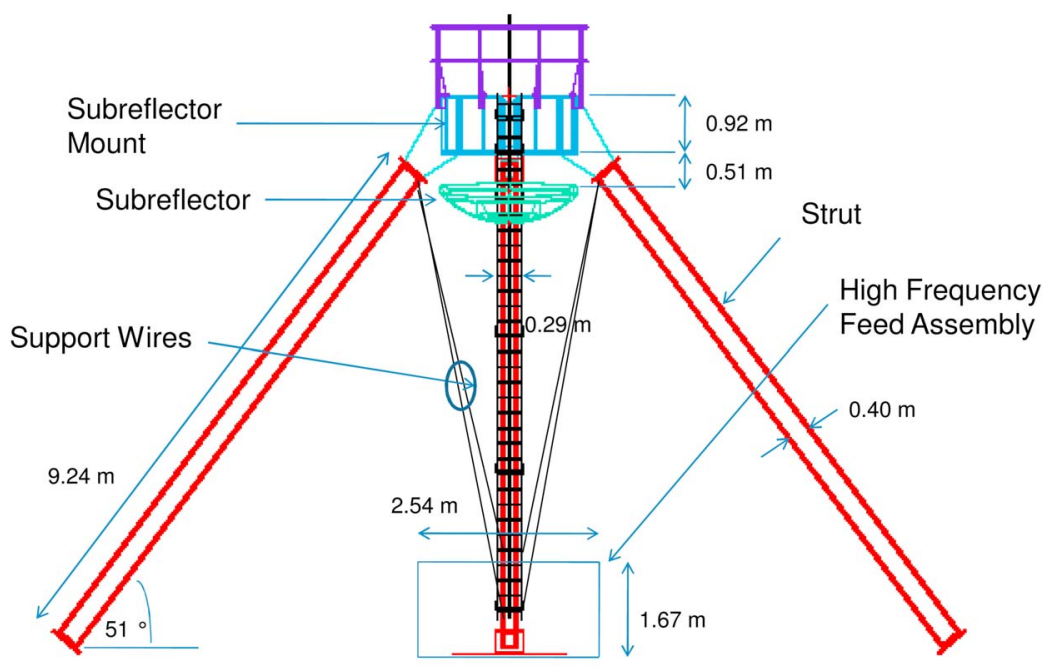

Figure 4. Detail of an EVLA reflector antenna geometry, showing the subreflector, the subreflector mount, the struts, the support wires and the feed assembly. The drawing is provided by J. Ruff of NRAO.

[24] The results of our analysis are shown in Figures 7 and 8 for the two possible orientations of the support wires. First, we consider the performance of the dipole which is co-aligned with the support wires (shown in Figure 7). Note the gain $(G)$ at $74 \mathrm{MHz}$ is predicted to be $20.4 \mathrm{~dB}$ which corresponds to an aperture efficiency of $29 \%$. The aperture efficiency, $\epsilon_{a}$ is calculated as the ratio of the effective aperture, $A_{e}\left(A_{e}=G \lambda^{2} / 4 \pi\right)$ and the physical aperture $\left(\pi D^{2} / 4\right)$ of the antenna. The gain of the cross-aligned dipole (Figure 8) at $74 \mathrm{MHz}$ is found to be $16.2 \mathrm{~dB}$, i.e., $4 \mathrm{~dB}$ worse, corresponding to $\epsilon_{a}=11 \%$. This is apparently due to an unfavorable interaction with the support wires in this configuration. This difference between the co- and cross-aligned dipoles has been observed in practice, but is difficult to quantify (F. N. Owen, National Radio Astronomy Observatory, private communication, September 2010). It is interesting to note that our estimates of $29 \%$ and $11 \%$ are consistent with the estimates of $25 \%$ and $15 \%$ reported by Kassim et al. [1993] and Kassim et al. [2007], respectively, although it is not clear if the latter pertains to different feed dipole orientations, as considered here.

[25] Also visible in Figures 7 and 8 is the fact that the pattern of the antenna system has slight asymmetry. This is because the subreflector is tilted as described in section 2 .

[26] The consistency of the simulated results with what is observed in practice gives us confidence in the model. Using

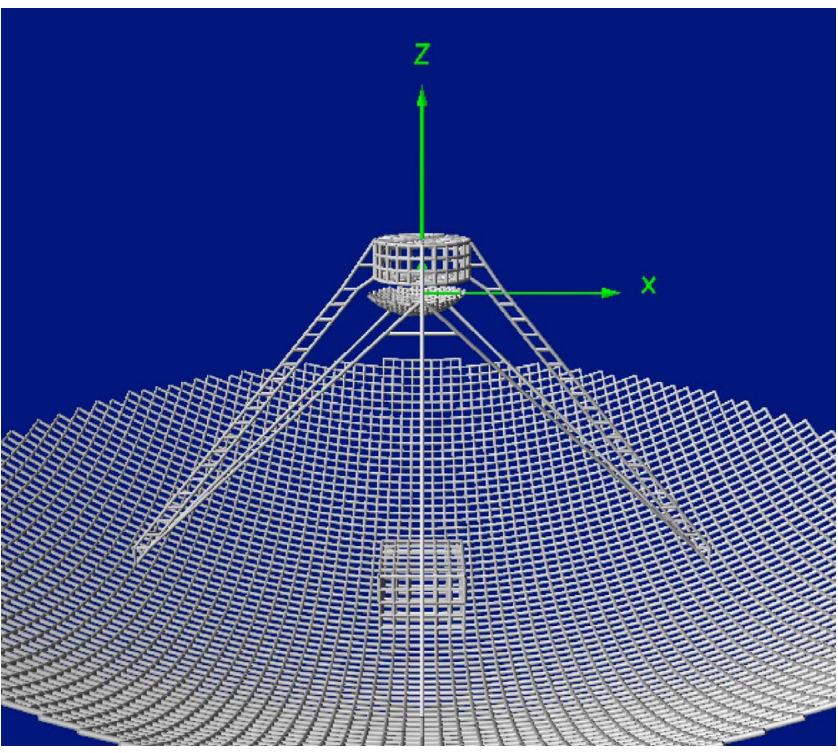

Figure 5. Orientation of the support wires in the EVLA antenna model with respect to a single co-aligned dipole feed.

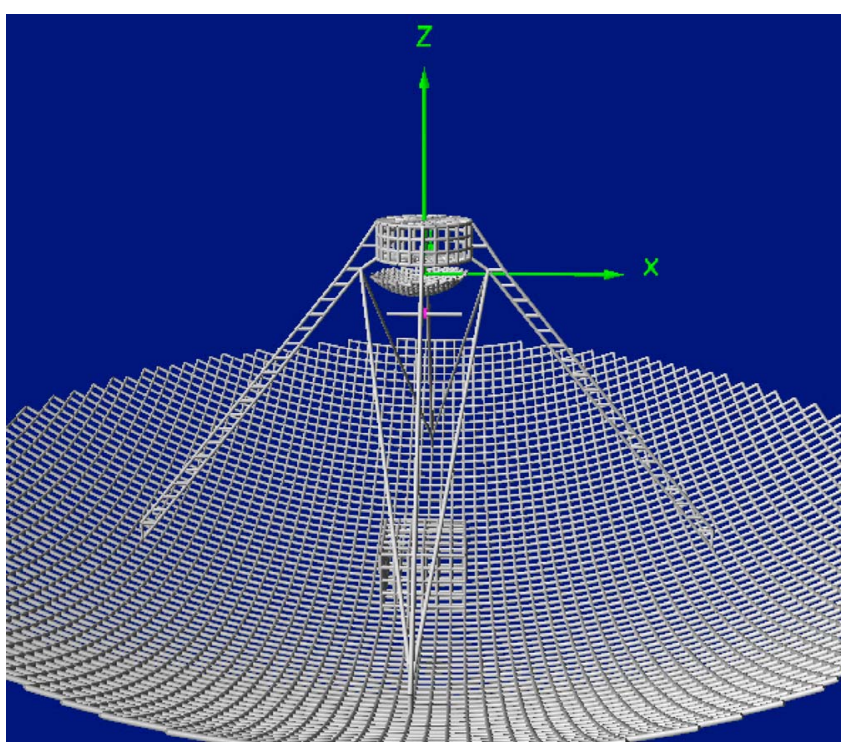

Figure 6. Orientation of the support wires in the EVLA antenna model with respect to a single cross-aligned dipole feed. 


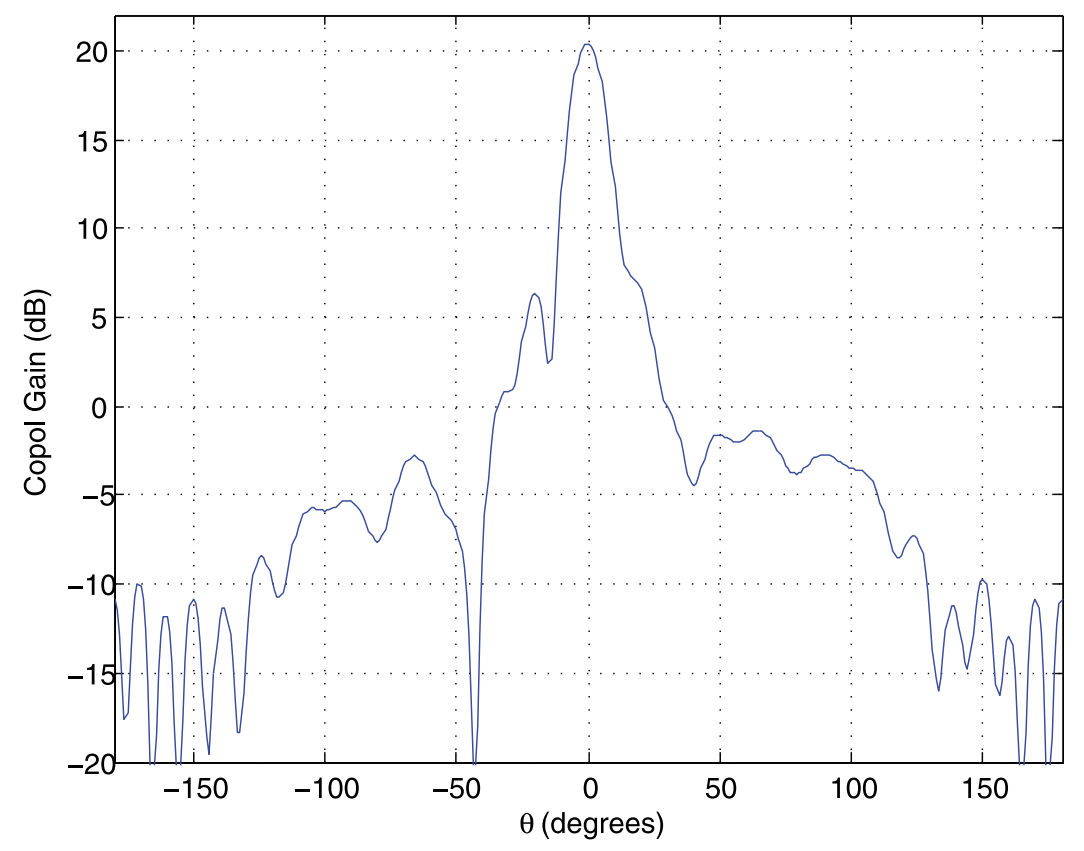

Figure 7. Calculated E-plane co-pol gain of the EVLA $4 \mathrm{~m}$ system for co-aligned support wires.

this model the sensitivities of an EVLA antenna for the existing $4 \mathrm{~m}$ system, and for the proposed new system, are analyzed in sections 4 and 5 , respectively.

\section{Analysis of Sensitivity of the Existing EVLA 4 m System}

[27] In this section, we estimate the sensitivity of the EVLA 4 m system in terms of SEFD. In order to compute the effective lengths of the feed dipoles, the complete reflector antenna model is illuminated with a $\theta$-polarized $1 \mathrm{~V} / \mathrm{m}$ plane wave incident from some direction $\psi$, and the resulting current $I_{L}$ across a series resistance $R_{L}$ at the feed terminals is determined using MoM. $a_{\theta}(\psi)$ for the feed antenna is then simply $I_{L} R_{L}$. The process is repeated for a $\phi$-polarized plane wave and iterated over $\psi$.

[28] The internal noise covariance matrix $\mathbf{P}_{u}$ is computed assuming that the internal noise between feed antennas is

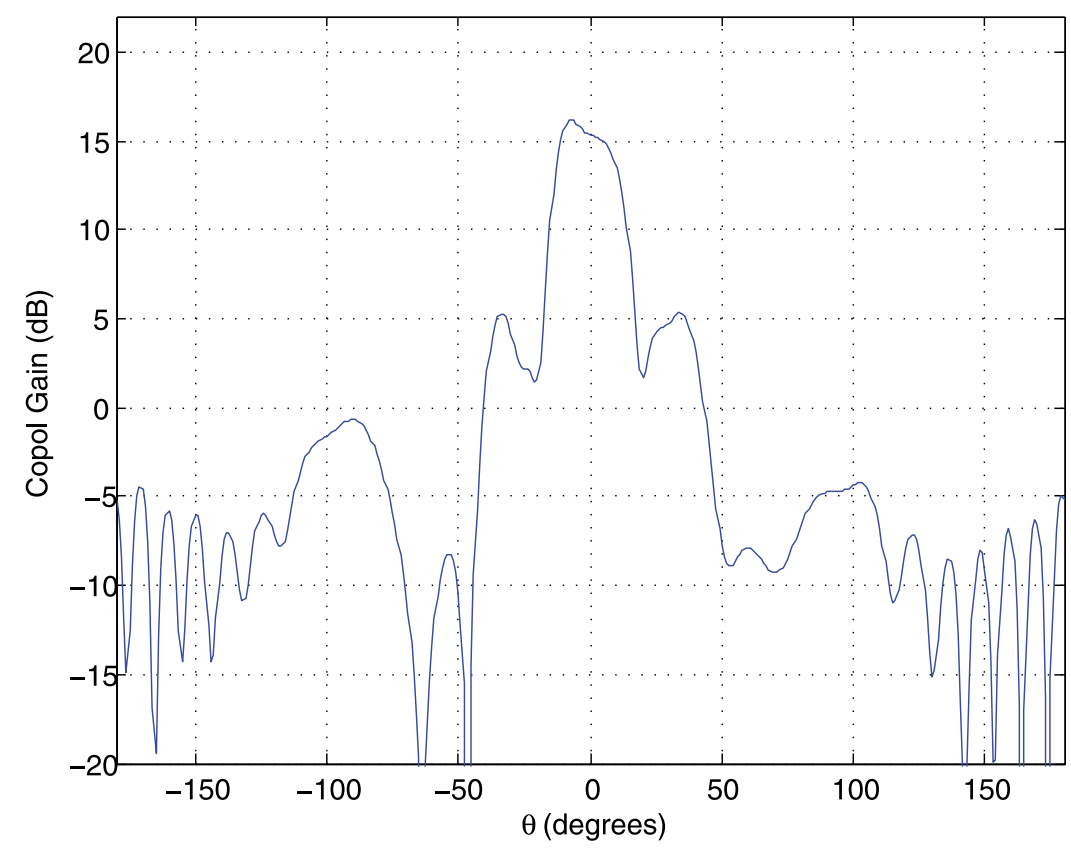

Figure 8. Calculated E-plane co-pol gain of the EVLA $4 \mathrm{~m}$ system for cross-aligned support wires. 


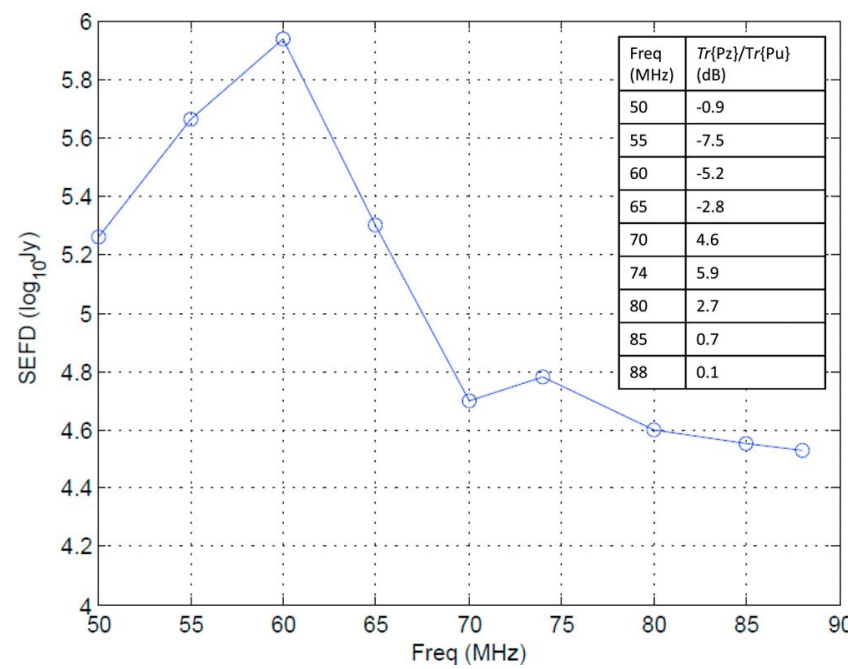

Figure 9. Estimated SEFD for the existing EVLA $4 \mathrm{~m}$ system. The inset table shows degree of Galactic noise dominance.

not correlated, so that $\mathbf{P}_{u}$ becomes a diagonal matrix whose non-zero elements are

$$
\mathbf{P}_{u}^{[n]}=k T_{p, n} R_{L}
$$

where $k$ is Boltzmann's constant $\left(1.38 \times 10^{-23} \mathrm{~J} / \mathrm{K}\right)$, and $T_{p, n}$ is the input-referred internal noise temperature associated with the $n$th antenna. In this paper, it is assumed that $R_{L}=$ $100 \Omega$, and $T_{p, n}=T_{p}=250 \mathrm{~K}$. These values are selected to be comparable to existing low frequency telescope systems that employ dipole-like antennas, e.g., the LWA [Ellingson et al., 2009], as opposed to the actual $4 \mathrm{~m}$ system receiver temperature, which is significantly higher.

[29] The external noise covariance matrix, $\mathbf{P}_{z}$ is computed using equation (20), where $T_{b}(\psi)$ is obtained using a model proposed by Ellingson [2005]. In this model $T_{b}(\psi)$ is

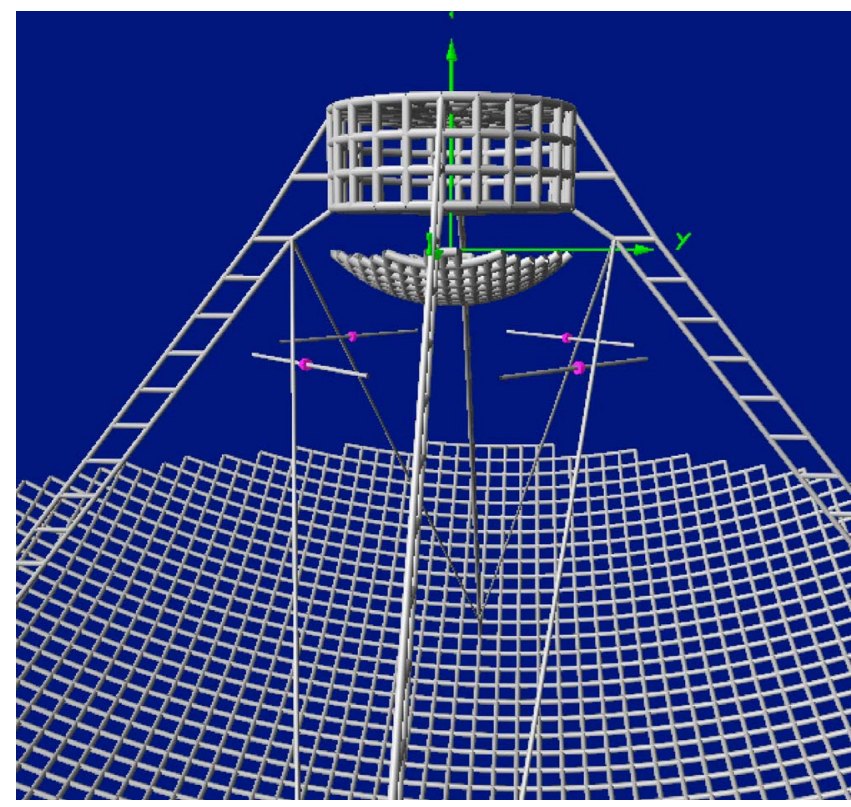

Figure 10. Single ring strut-straddling feed array. assumed to be uniform over the sky $(\theta<\pi / 2)$ and zero for $\theta>\pi / 2$. Using this model $T_{b}(\psi)$ toward the sky is found to be $4835 \mathrm{~K}, 3035 \mathrm{~K}, 1777 \mathrm{~K}$, and $1142 \mathrm{~K}$ at $50 \mathrm{MHz}$, $60 \mathrm{MHz}, 74 \mathrm{MHz}$ and $88 \mathrm{MHz}$ respectively. The actual contributions to system temperature are less due to the impedance mismatch between the antenna self-impedance and $R_{L}$. This mismatch is automatically taken into account in the way we determine the effective lengths of the feed antenna. The signals from the two crossed dipoles are combined to obtain circular polarization.

[30] The results of our analysis are shown in Figure 9. SEFD estimations for the system are calculated only in the range 50-88 MHz. Due to the presence of interference from the FM broadcast band, the range $88-100 \mathrm{MHz}$ is not considered in this paper. As can be seen from Figure 9, SEFD increases (i.e., becomes worse) below $70 \mathrm{MHz}$. The reason for this is the fact that the impedance mismatch starts to become so bad that the system no longer remains Galactic-noise limited, and the internal noise starts to define the sensitivity. The ratio of $\operatorname{Tr}\left\{\mathbf{P}_{z}\right\} / \operatorname{Tr}\left\{\mathbf{P}_{u}\right\}$ (where " $\operatorname{Tr}$ " denotes the trace operation; i.e., the sum of the diagonal terms.) expresses the degree by which Galactic noise dominates the internal noise at the combined output, and is shown in the inset of Figure 9.

\section{Strut-Straddling Feed Array}

[31] In this section, we consider the strut-straddling feed array. In this arrangement the dipoles are mounted in a ringlike formation between adjacent struts as shown in Figure 10. The radii of these dipoles are selected to be $4.76 \mathrm{~mm}$, and their lengths are determined to make them resonant at $74 \mathrm{MHz}$. The radius is increased from that of the existing dipoles $(2.38 \mathrm{~mm})$ in order to improve the impedance bandwidth; so that we can achieve Galactic noise-limited sensitivity over a wider tuning range without making major changes to the shape and size of the feed. The signal path is modeled such that the signals from the dipoles and their associated electronics are multiplied by combining coefficients that maximize the SNR, and the products are summed into a single

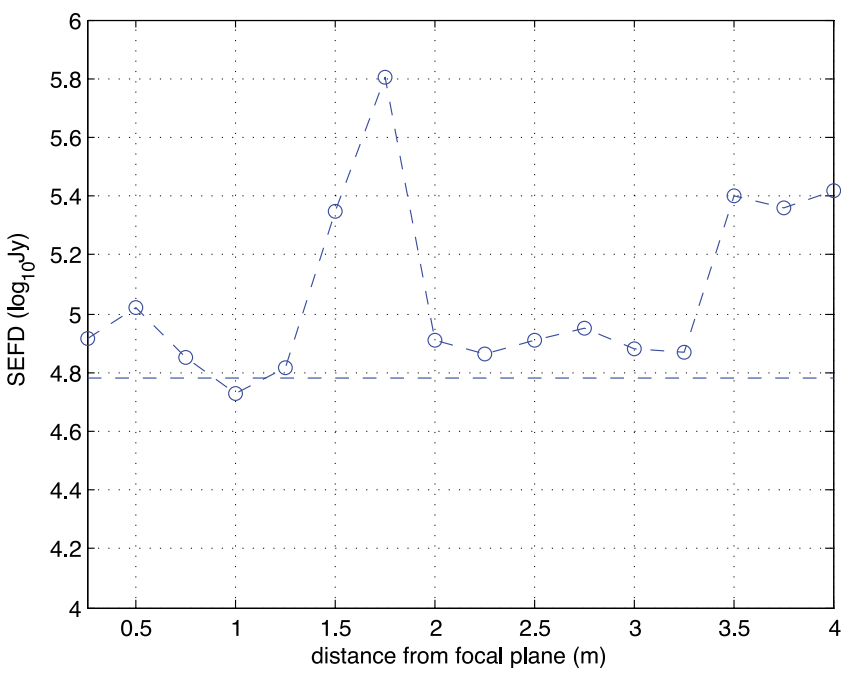

Figure 11. Location selection for mounting strut-straddling feed array based on SEFD at $74 \mathrm{MHz}$. Dashed line is the SEFD of the existing EVLA $4 \mathrm{~m}$ system at $74 \mathrm{MHz}$. 


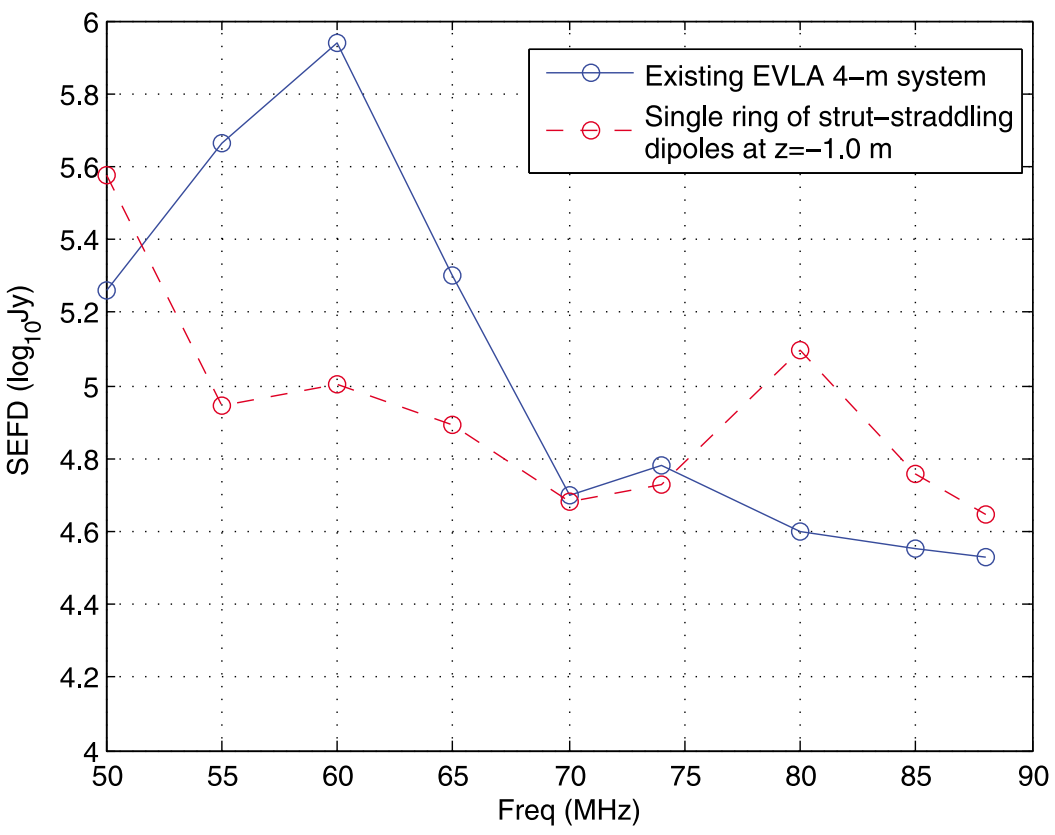

Figure 12. Estimated SEFD for a single ring of strut-straddling dipoles at $z=-1.0 \mathrm{~m}$, and comparison with the existing EVLA 4 m system.

output. To properly consider the broadband performance, the combining coefficients are recomputed at each frequency of interest.

[32] A key design parameter in this scheme is the location of the plane in which the ring of dipoles will be located. We considered planes from $z=-0.25 \mathrm{~m}$ to $z=-4.0 \mathrm{~m}$ in increments of $0.25 \mathrm{~m}$. The study is limited within these locations due to the huge computational burden involved with the analysis (a single-frequency simulation requires about 3 hours in a $2.33 \mathrm{GHz}$ quad-core processor machine). In each of these planes we employed the ring of dipoles, and calculated the SEFD at $74 \mathrm{MHz}$. The results are shown in Figure 11. The lowest SEFD at $74 \mathrm{MHz}$ is observed for dipoles in the plane $z=-1.0 \mathrm{~m}$ at which the SEFD is within $10 \%$ of that for the existing $4 \mathrm{~m}$ system. This location is therefore selected as the preferred location for mounting a single ring of strut-straddling dipoles. The second lowest SEFD is observed at $z=-2.25 \mathrm{~m}$, and is comparably low. This suggests the possible utility of a second ring of dipoles.

[33] The SEFD for the new system with a single ring of dipoles at $z=-1.0 \mathrm{~m}$ are estimated and compared with the EVLA $4 \mathrm{~m}$ system. The results are shown in Figure 12.

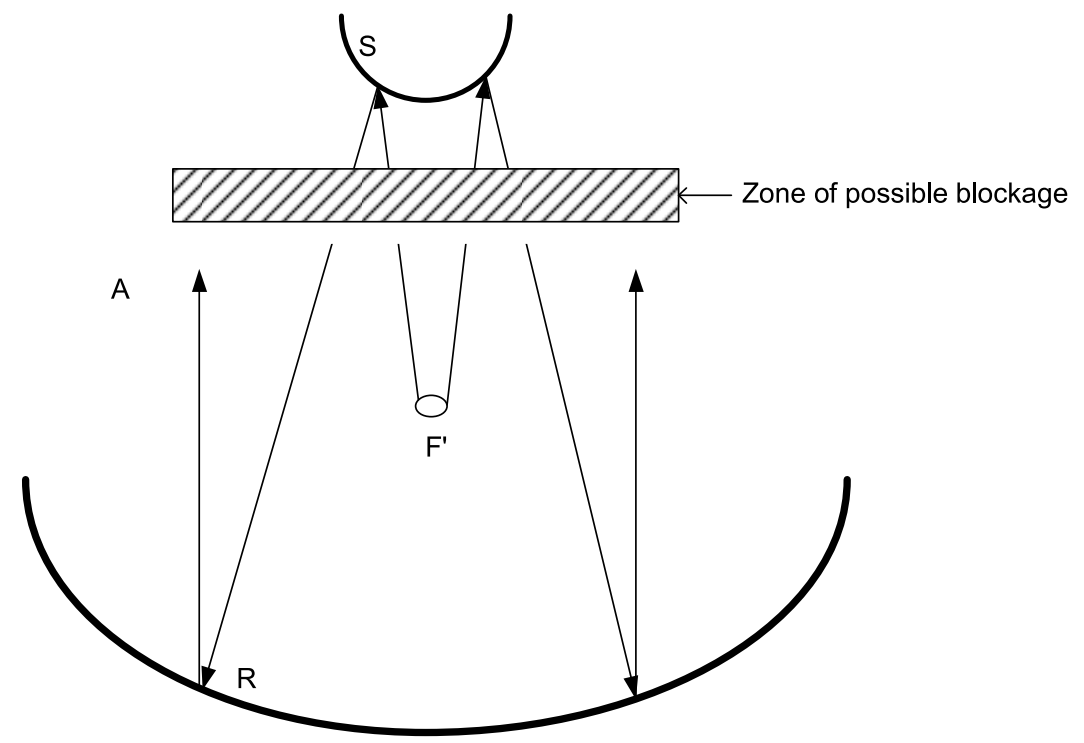

Figure 13. Propagation paths in a Cassegrain reflector antenna in transmit mode, corresponding to EVLA operation at wavelengths of $21 \mathrm{~cm}$ and below. 


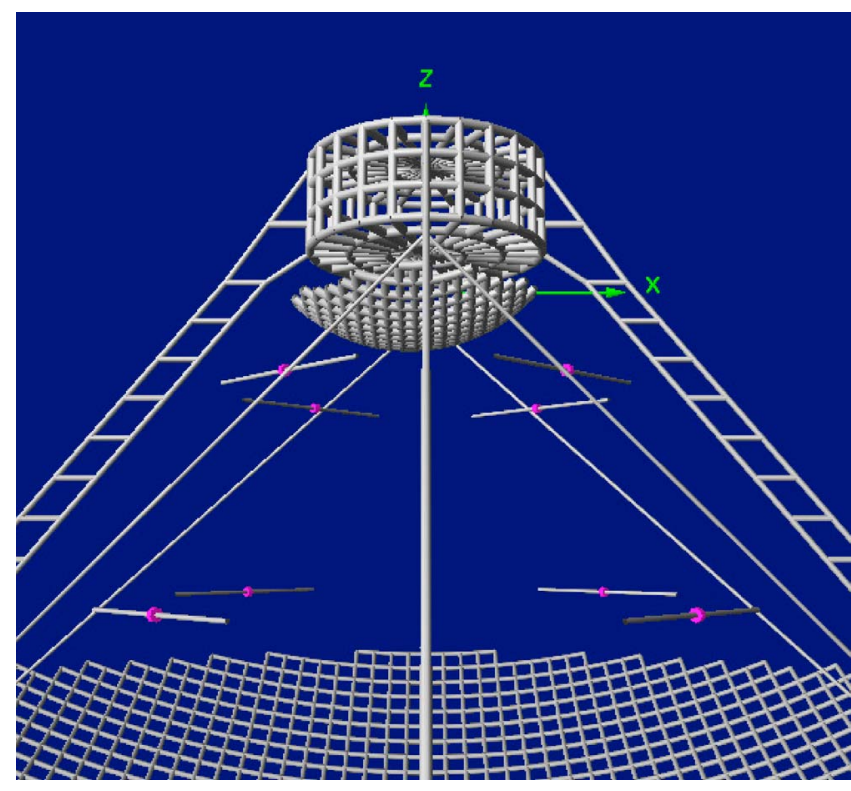

Figure 14. Double ring strut-straddling feed array.

It can be observed that the new system has comparable performance to the existing system; and in the range $55-65 \mathrm{MHz}$ the new system performs better. At $74 \mathrm{MHz}$ the new system yields $\mathrm{SEFD} \approx 53 \mathrm{kJy}$ whereas the existing system yields $\mathrm{SEFD} \approx 60 \mathrm{kJy}$.

[34] In order to quantify the blocking by the dipole feeds, we first identify three different paths of propagation in a Cassegrain reflector antenna, corresponding to EVLA operation at wavelengths of $21 \mathrm{~cm}$ or below. This is shown in Figure 13. The first path $\overline{F^{\prime} S}$ is from the secondary focus to the subreflector. The second path $\overline{S R}$ is from the subreflector to the main reflector. The third path $\overline{R A}$ is from the main reflector toward the far field. In order to estimate the blockage in the first two paths, the field incident on the main reflector due to a feed located at the secondary focus is calculated using MoM. The analysis is performed separately for the cases when blocking dipoles are present at the image of the prime focus, and when the dipoles are mounted between adjacent struts. For both of these cases, the resulting physical optics currents on the reflector surface are calculated, and then radiated to find the directivity of the system. When the dipoles located at the image of the prime focus are replaced with a single ring of strut-straddling dipoles at $z=$ $-1.0 \mathrm{~m}$, the effective aperture is seen to increase by $3.7 \%$ at $1.4 \mathrm{GHz}$. Therefore, blockage at $1.4 \mathrm{GHz}$ is significantly reduced in the paths $\overline{F^{\prime} S}$ and $\overline{S R}$ when the new feed system is employed.

[35] In the above analysis blockage in the path $\overline{R A}$ is not considered. In order to quantify blockage in this path at $1.4 \mathrm{GHz}$, we need to compute the fields reflected from the main reflector, upon the excitation of a feed located at the secondary focus, and the interaction of these fields with the scattering objects (struts, subreflector, blocking dipoles) present in front of the main reflector. This requires a full wave analysis at $1.4 \mathrm{GHz}$, which is not practical. Therefore, it is difficult to accurately quantify blockage in the path $\overrightarrow{R A}$. However, an approximate study to compare the blockage in this path due to the strut-straddling dipoles and the image prime focus dipoles is possible. In this study, we illuminate the entire structure in front of the main reflector, but in the absence of the main reflector, with an uniform plane wave incident from the negative $z$-axis, both for the cases when the blocking dipoles are present at the image of the prime focus, and when the dipoles are mounted between adjacent struts. The scattering cross section in the forward direction is

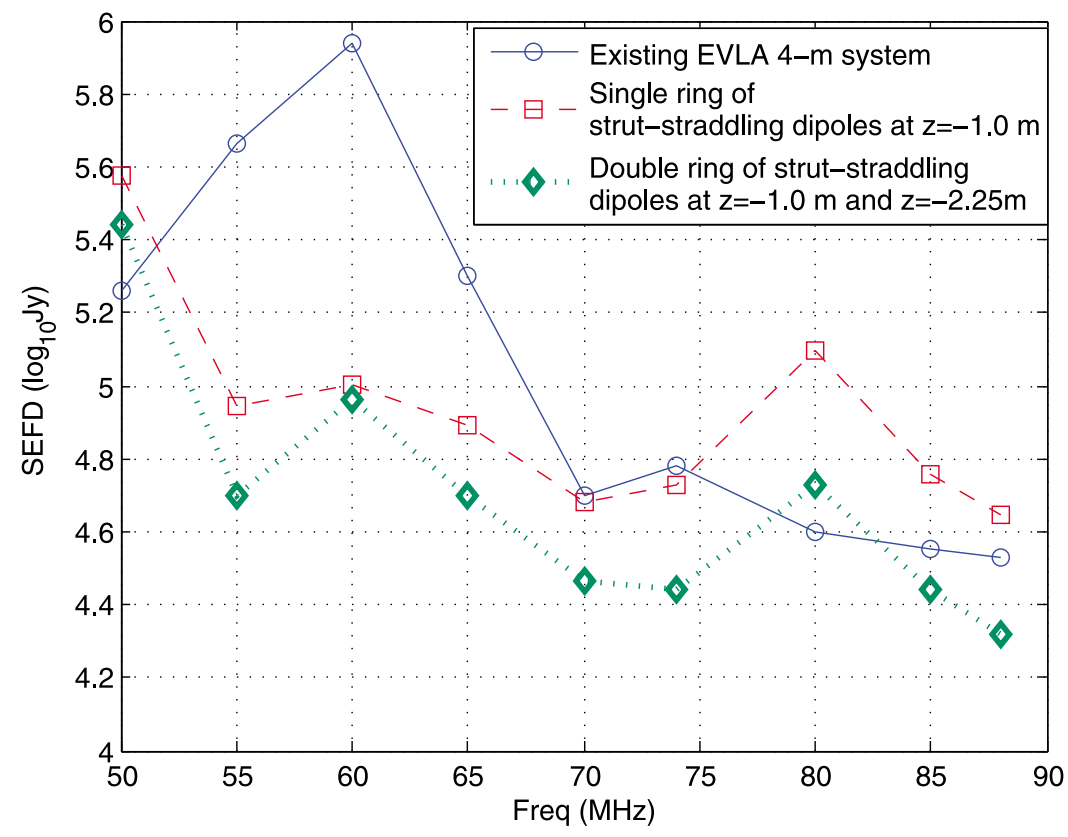

Figure 15. Estimated SEFD for double ring of strut-straddling dipoles. 
Table A1. 3-D Coordinates and the Beam-Forming Coefficients for the Single Ring of Dipoles at $z=-1.0 \mathrm{~m}$

\begin{tabular}{lcccc}
\hline & Dipole 1 & Dipole 2 & Dipole 3 & Dipole 4 \\
\hline 3-D coordinates $(\mathrm{m}):$ end1 $(x, y, z)$ & $(0.52,1.90,-1.00)$ & $(-1.90,-0.52,-1.00)$ & $(-0.52,1.90,-1.00)$ & $(1.90,-0.52,-1.00)$ \\
3-D coordinates $(\mathrm{m}):$ end2 $(x, y, z)$ & $(1.90,0.52,-1.00)$ & $(-0.52,-1.90,-1.00)$ & $(-1.90,0.52,-1.00)$ & $(0.52,-1.90,-1.00)$ \\
$b_{n}$ at $50 \mathrm{MHz}$ & $-0.5+0.1 j$ & $-0.5+0.0 j$ & $-0.5+0.1 j$ & $-0.5+0.0 j$ \\
$b_{n}$ at $55 \mathrm{MHz}$ & $-0.5+0.0 j$ & $-0.5+0.1 j$ & $-0.5+0.0 j$ & $-0.5+0.1 j$ \\
$b_{n}$ at $60 \mathrm{MHz}$ & $-0.5-0.1 j$ & $-0.5+0.0 j$ & $0.5+0.1 j$ & $0.5+0.0 j$ \\
$b_{n}$ at $65 \mathrm{MHz}$ & $-0.5-0.1 j$ & $-0.5+0.0 j$ & $0.5+0.1 j$ & $0.5+0.0 j$ \\
$b_{n}$ at $70 \mathrm{MHz}$ & $-0.5+0.0 j$ & $-0.5+0.0 j$ & $-0.5+0.0 j$ & $-0.5+0.0 j$ \\
$b_{n}$ at $74 \mathrm{MHz}$ & $0.5+0.1 j$ & $0.5+0.0 j$ & $-0.5+0.1 j$ & $-0.5+0.0 j$ \\
$b_{n}$ at $80 \mathrm{MHz}$ & $-0.5-0.1 j$ & $-0.5+0.0 j$ & $0.5+0.1 j$ & $-0.5+0.0 j$ \\
$b_{n}$ at $85 \mathrm{MHz}$ & $-0.5+0.0 j$ & $-0.5+0.1 j$ & $-0.5+0.0 j$ & $-0.5+0.1 j$ \\
$b_{n}$ at $88 \mathrm{MHz}$ & $-0.5+0.0 j$ & $-0.5+0.0 j$ & $-0.5+0.0 j$ & $-0.5+0.0 j$ \\
\hline
\end{tabular}

then calculated. It is observed that when the dipoles located at the image of the prime focus are replaced with the strutstraddling dipoles at $z=-1.0 \mathrm{~m}$, the scattering cross section increases by about $3 \%$. However, in this case the scattering cross section is computed assuming an uniform plane wave, whereas in practice a non-uniform plane wave is produced upon reflection from the main reflector due to the spherical spreading of the feed pattern incident on it. In fact, the power density of the field reflected from the reflector surface is at least $3 \%$ higher along the $z$-axis than at the location of the strut-straddling dipoles. The increased blockage due to increased scattering from the strut-straddling dipoles is approximately canceled by the reduced power density of the incident field which they scatter. Thus, both feed systems introduce about the same blockage at $1.4 \mathrm{GHz}$ along the $\overline{R A}$ path.

[36] Taking into account both the $\overline{F^{\prime} S R}$ and $\overline{R A}$ paths, we estimate a reduction in blockage from about $6 \%$ (as estimated by Kassim et al. [1993]) for the existing $4 \mathrm{~m}$ system to about $2.3 \%$ at $1.4 \mathrm{GHz}$ using the strut-straddling system.

[37] A possible way to improve the sensitivity obtained with a single ring of strut-straddling dipoles without making blockage worse is to use two rings, as shown in Figure 14. As pointed out earlier, $z=-1.0 \mathrm{~m}$ and $z=-2.25 \mathrm{~m}$ appear to be the suitable choices for the locations of the two rings. Figure 15 shows the results for this arrangement. Again, the combining coefficients are selected to maximize SNR. Note that the SEFD for the double ring system is better than the existing system at all the frequencies except for 50 and $80 \mathrm{MHz}$. Again, in the same manner as described above, the blockage introduced by this system at $1.4 \mathrm{GHz}$ is estimated. There is not any significant increase (less than $0.2 \%$ ) in blocking in the path $\overline{F^{\prime} S R}$ when the single ring of dipoles is replaced by the double ring. Also, in the path $\overline{R A}$ the increase in blockage is estimated to be negligible as the scattering cross section in the forward direction for the double ring is increased only by $4 \%$ from that of the single ring; whereas the incident power density in the location of the second ring $(z=-2.25 \mathrm{~m})$ is at least $4 \%$ less than that at the location of the first ring $(z=-1.0 \mathrm{~m})$.

[38] For design optimization purposes, it would be useful to have a simple rule or guidelines for choosing the locations for rings of dipoles. Unfortunately, this does not appear to be possible, due to the complex interactions between the dipoles and between the dipoles and the dish structure. At present, this optimization appears to be possible only by trial and error.

[39] The strut-straddling feed arrays described above demonstrated performance comparable to the existing system, but with reduced blockage. An issue of concern may be the support structure for the dipoles and the blockage associated with this support. The dipoles of strut-straddling arrays can be easily mounted and supported using nonconducting rope between the struts. Also, the cables carrying the signals from the dipoles can run along these ropes to the struts and then be brought along the struts to the back of the subreflector where they can be combined. In order to estimate the effect of the cables, we did a simple study where one end of each of the dipoles is extended to the nearest struts to model the cable, and the blocking in the path $\overline{F^{\prime} S R A}$ is again computed. It is found that there is no significant increase (less than $0.2 \%$ ) in blockage in the $\overline{F^{\prime} S R}$ path for extending the dipoles. On the other hand, the scattering cross section in the forward direction is increased

Table A2. 3-D Coordinates and the Beam-Forming Coefficients for the Dipole Ring at $z=-1.0 \mathrm{~m}$ in the Double Ring Arrangement

\begin{tabular}{lcccc}
\hline & Dipole 1 & Dipole 2 & Dipole 3 & Dipole 4 \\
\hline 3-D coordinates $(\mathrm{m}):$ end1 $(x, y, z)$ & $(0.52,1.90,-1.00)$ & $(-1.90,-0.52,-1.00)$ & $(-0.52,1.90,-1.00)$ & $(1.90,-0.52,-1.00)$ \\
3-D coordinates $(\mathrm{m})$ : end2 $(x, y, z)$ & $(1.90,0.52,-1.00)$ & $(-0.52,-1.90,-1.00)$ & $(-1.90,0.52,-1.00)$ & $(0.52,-1.90,-1.00)$ \\
$b_{n}$ at $50 \mathrm{MHz}$ & $0.4-0.1 j$ & $0.4-0.1 j$ & $0.2-0.1 j$ & $0.5+0.0 j$ \\
$b_{n}$ at $55 \mathrm{MHz}$ & $-0.3+0.3 j$ & $-0.2+0.3 j$ & $0.4+0.1 j$ & $0.3+0.1 j$ \\
$b_{n}$ at $60 \mathrm{MHz}$ & $-0.4-0.1 j$ & $-0.5+0.0 j$ & $0.0+0.0 j$ & $0.5+0.0 j$ \\
$b_{n}$ at $65 \mathrm{MHz}$ & $0.0+0.4 j$ & $0.1+0.4 j$ & $0.1+0.0 j$ & $0.1-0.1 j$ \\
$b_{n}$ at $70 \mathrm{MHz}$ & $0.0+0.3 j$ & $0.0+0.3 j$ & $-0.2+0.0 j$ & $0.3-0.1 j$ \\
$b_{n}$ at $74 \mathrm{MHz}$ & $0.4-0.0 j$ & $0.5-0.0 j$ & $0.0+0.0 j$ & $-0.2-0.1 j$ \\
$b_{n}$ at $80 \mathrm{MHz}$ & $-0.3+0.1 j$ & $-0.3+0.2 j$ & $-0.1+0.0 j$ & $-0.1+0.0 j$ \\
$b_{n}$ at $85 \mathrm{MHz}$ & $-0.2+0.0 j$ & $-0.4-0.1 j$ & $0.0-0.1 j$ & $0.1+0.1 j$ \\
$b_{n}$ at $88 \mathrm{MHz}$ & $-0.4-0.1 j$ & & &
\end{tabular}


Table A3. 3-D Coordinates and the Beam-Forming Coefficients for the Dipole Ring at $z=-2.25 \mathrm{~m}$ in the Double Ring Arrangement

\begin{tabular}{lcccc}
\hline & Dipole 1 & Dipole 2 & Dipole 3 & Dipole 4 \\
\hline 3-D coordinates $(\mathrm{m}):$ end1 $(x, y, z)$ & $(1.00,2.40,-2.25)$ & $(-2.40,-1.00,-2.25)$ & $(-1.00,2.40,-2.25)$ & $(2.40,-1.00,-2.25)$ \\
3-D coordinates $(\mathrm{m})$ : end2 $(x, y, z)$ & $(2.40,1.00,-2.25)$ & $(-1.00,-2.40,-2.25)$ & $(-2.40,1.00,-2.25)$ & $(1.00,-2.40,-2.25)$ \\
$b_{n}$ at $50 \mathrm{MHz}$ & $0.1+0.1 j$ & $0.1+0.1 j$ & $0.5+0.0 j$ & $0.2+0.0 j$ \\
$b_{n}$ at $55 \mathrm{MHz}$ & $-0.1-0.2 j$ & $-0.1-0.3 j$ & $0.5+0.0 j$ & $0.0+0.2 j$ \\
$b_{n}$ at $60 \mathrm{MHz}$ & $-0.1+0.0 j$ & $0.0+0.0 j$ & $0.2+0.1 j$ & $0.0-0.1 j$ \\
$b_{n}$ at $65 \mathrm{MHz}$ & $0.5+0.0 j$ & $0.5+0.0 j$ & $0.4+0.0 j$ & $0.1-0.3 j$ \\
$b_{n}$ at $70 \mathrm{MHz}$ & $0.3-0.1 j$ & $0.2-0.2 j$ & $-0.6+0.0 j$ & $-0.1-0.4 j$ \\
$b_{n}$ at $74 \mathrm{MHz}$ & $-0.3-0.1 j$ & $-0.3-0.1 j$ & $0.1+0.1 j$ & $-0.1-0.1 j$ \\
$b_{n}$ at $80 \mathrm{MHz}$ & $0.6+0.0 j$ & $0.6+0.0 j$ & $0.0+0.2 j$ & $0.0-0.1 j$ \\
$b_{n}$ at $85 \mathrm{MHz}$ & $0.6+0.0 j$ & $0.6+0.0 j$ & $0.0+0.3 j$ & $0.3-0.1 j$ \\
$b_{n}$ at $88 \mathrm{MHz}$ & $0.5+0.0 j$ & $0.5+0.0 j$ & & $-0.1-0.1 j$ \\
\hline
\end{tabular}

by about $1.5 \%$ when the dipoles are extended, and thus might slightly increase the blockage. Nevertheless, the strutstraddling dipoles with their supports are still estimated to reduce the blockage at $1.4 \mathrm{GHz}$ relative to the image-prime focus system.

\section{Conclusion}

[40] The performance of the existing EVLA $4 \mathrm{~m}$ system is characterized at frequencies below $100 \mathrm{MHz}$ in terms of SEFD. In order to overcome the blockage associated with the existing feed system we proposed strut-straddling feed arrays. Strut-straddling feed arrays demonstrated performance comparable to or better than the existing image prime focus system in the frequency range about 50-90 $\mathrm{MHz}$. Furthermore, our results indicate that this system achieves a significant reduction in blockage at $1.4 \mathrm{GHz}$ (wavelength of $21 \mathrm{~cm}$ ), possibly facilitating permanent installation.

[41] Although the focus of this paper has been the EVLA, the design technique and analysis methodology described in this paper should be applicable to other large reflector antennas as well.

[42] The implementation described in this paper produces a single polarization. Future work includes development of combining schemes to obtain dual orthogonal polarizations.

\section{Appendix A: Beam-Forming Coefficients and Dipole Locations}

[43] The beam-forming coefficients and the 3-D spatial coordinates for the single ring of dipoles at $z=-1.0 \mathrm{~m}$ are given in Table A1. Also, the beam-forming coefficients and the 3-D spatial coordinates for the double ring of dipoles at $z=-1.0 \mathrm{~m}, z=-2.25 \mathrm{~m}$ are given in Tables A2 and A3, respectively.

\section{References}

Burke, G., and A. Poggio (1981), Numerical Electromagnetics Code (NEC) Method of Moments, Part III: User's Guide, Lawrence Livermore Lab, Livermore, Calif.

Ellingson, S. W. (2005), Antennas for the next generation of low-frequency radio telescopes, IEEE Trans. Antennas Propag., 53(8), 2480-2489, doi:10.1109/TAP.2005.852281.

Ellingson, S. W. (2011), Sensitivity of antenna arrays for long-wavelength radio astronomy, IEEE Trans. Antennas Propag., 59(6), 1855-1863, doi:10.1109/TAP.2011.2122230.

Ellingson, S. W., J. H. Simonetti, and C. D. Patterson (2007), Design and evaluation of an active antenna for a $29-47 \mathrm{MHz}$ radio telescope array, IEEE Trans. Antennas Propag., 55(3), 826-831, doi:10.1109/ TAP.2007.891866.

Ellingson, S. W., et al. (2009), The long wavelength array, Proc. IEEE, 97(8), 1421-1430, doi:10.1109/JPROC.2009.2015683.

Harun, M., and S. W. Ellingson (2008), Analysis of a dipole-fed VLA dish below $100 \mathrm{MHz}$, technical report, Virginia Polytechnic Institute and State University.

Kassim, N. E., et al. (1993), Subarcminute resolution imaging of radio sources at $74 \mathrm{MHz}$ with the very large array, Astron. J., 106, 2218-2228, doi:10.1086/116795.

Kassim, N. E., et al. (2007), The $74 \mathrm{MHz}$ system on the very large array, Astrophys. J. Suppl. Ser., 172, 686-719, doi:10.1086/519022.

Monzingo, R. A., and T. W. Miller (1980), Introduction to Adaptive Arrays, John Wiley, New York.

Napier, P. J., et al. (1983), The very large array: Design and performance of a modern synthesis radio telescope, Proc. IEEE, 71(11), 1295-1320, doi:10.1109/PROC.1983.12765.

Olsson, R., et al. (2006), The Eleven antenna: A compact decade bandwidth dual polarized feed for reflector antennas, IEEE Trans. Antennas Propag., 54(2), 368-375, doi:10.1109/TAP.2005.863392.

Ott, J., et al. (2009), Pushing the limits of the EVLA: An enhancement program for the next decade, Astro2010 activity white paper, Natl. Acad. of Sci., Washington, D. C. [Available at http://sites.nationalacademies.org/ BPA/BPA_049855.]

Rubinstein, A., F. Rachidi, and M. Rubinstein (2005), On wire-grid representation of solid metallic surfaces, IEEE Trans. Electromagn. Compat., 47(1), 192-195, doi:10.1109/TEMC.2004.838230.

Shankar, N. U., et al. (2009), A $50 \mathrm{MHz}$ system for GMRT, in The LowFrequency Radio Universe, edited by D. J. Saikia, D. A. Green, and Y. Gupta, Astron. Soc. Pac. Conf. Ser., 407, 393-397.

van der Marel, J., et al. (2005), Low frequency receivers for the WSRT: A window of opportunity, in Proceedings of URSI-GA '05, Int. Union of Radio Sci., New Delhi, India. [Available at http://www.ursi.org/ Proceedings/ProcGA05/pdf/J03-P.14(0817).pdf.]

S. W. Ellingson and M. Harun, Bradley Department of Electrical and Computer Engineering, Virginia Tech, 432 Durham, Blacksburg, VA 24060 , USA. (ellingson@vt.edu; mharun@vt.edu) 